\title{
MUJER, EROTISMO Y SEXUALIDAD EN EL QUIJOTE
}

La importancia de los estudios sobre la presencia de la mujer en la literatura del Siglo de Oro y otros aspectos relacionados ha alcanzado en fechas recientes cotas elevadas: se ha convertido en un campo de trabajo muy tratado por la crítica, al que se han dedicado ya estudios numerosos, realizados desde perspectivas y con intereses distintos.

Con Cervantes y, en particular, con el Quijote, se ha producido un fenómeno similar'. A ello responde, por ejemplo, la antología de textos cervantinos publicada en 1981 con el título Amor $y$ sexo en Cervantes ${ }^{2}$ y la celebración de dos congresos que han abordado estos temas.

En efecto, el IX Seminario Internacional sobre Literatura Española y Edad de Oro, que se celebró en la primavera de 1989 en la Universidad Autónoma de Madrid, tuvo como título general El erotismo y la literatura clásica española. En él expresaron sus opiniones sobre estos aspectos en relación con el Quijote los profesores Carroll B. Johnson, Monique Joly y Agustín Redondo. Sus aportaciones pueden leerse ahora en el volumen IX de la revista Edad de Oro. Dos años más tarde, en noviembre y diciembre de 1991, se celebró en Montilla el coloquio El erotismo y la brujería en Cervantes, con intervenciones sobre el Quijote de Monique Joly

I Estudios ya lejanos, pero todavía interesantes y valiosos son los de RAmiro De Maeztu, Don Quijote o el amor [1926]. Edición de Alberto Sánchez. Salamanca, Anaya, 1964; y CONCHA EsPINA, «Mujeres del Quijote», Obras completas de Concha Espina. Madrid, Ediciones Fax, 1955, 2." ed., vol. II, pp. 826-52.

2 Vid. Roma Marieu (sel. y ed.), Amor y sexo en Cervantes. Madrid, Altalena, 1981. 
y Alfredo Baras Escolá. Sus trabajos pueden encontrarse ahora en el volumen XII, 2 (Fall, 1992) de la revista Cervantes.

Se ha pasado, pues, de un período en el que estos temas se consideraban casi como tabú, a otro en el que no sólo se han puesto de moda, sino que han dado lugar a análisis o interpretaciones de la obra cervantina que se alejan demasiado del propio texto y, además, no siempre ayudan a entender lo escrito por el propio autor. Monique Joly se ha referido, con certeras palabras, a este

«[...] movimiento a raíz del cual se habia puesto de moda hablar de realidades cuya existencia ni siquiera se reconocía, unos decenios antes, en los ambientes académicos en los que se estaba ahora promoviendo su estudio. Confieso que consideraba el impulso que favorecía a este tema de estudio tan cuestionable como la ceguera o la mojigatería de la época anterior. Fue por eso [...] un motivo de satisfacción escuchar de labios de una persona tan autorizada como el profesor Jammes unas reservas parecidas a las mías, al referirse a la ceguera y a los excesos que en sentido inverso se habían cometido, como los Escilas y Caribdis de la investigación en este delicado terreno; ${ }^{3}$.

Acertadas palabras, sí, pues este movimiento o línea de investigación sobre el Quijote ha proporcionado, en efecto, trabajos importantes, iluminadores, en ocasiones, de distintos aspectos o episodios de la novela cervantina; pero, al mismo tiempo, ha dado lugar a afirmaciones encontradas, difíciles de conciliar las unas con las otras. $\mathrm{Y}$ así, por ejemplo, mientras Rainer Rutkowski, en interpretación muy reductora de la novela, ve el Quijote como obra esencialmente misógina y antifeminista ${ }^{4}$; Héctor P. Márquez, en extenso libro que, sin embargo, no ofrece novedad alguna sobre estos temas y, a la vez, presenta algunas contradicciones y simplificaciones inexplicables ${ }^{5}$, define a

${ }^{3}$ MONIQUe Joly, «Erotismo y marginación social en la novela cervantina», Cervantes, XII, 2, 1992, p. 8.

- Rainer RUtKowski, "Misoginia y nostalgia en las escenas bucolicas del Quijotex, Cuadernos Hispanoamericanos, 430, abril, 1986, pp. 61-3.

5 Héctor P. MARquez, La representación de los personajes femeninos en el "Quijote", Madrid, José Porrúa Turanzas, 1990. En p. 182 se puede leer: «En realidad no resultaron personajes femeninos verdaderamente sobresalientes, y no hacen falta en la novela porque los protagonistas son seres completos que dominan la acción. Pero se tiene que reconocer que sin las mujeres la novela no llegaría al nivel que alcanzó simplemente porque la acción fundamental no serfa igual sin ellas. Además de Dulcinea, todas las figuras femeninas, que son más numerosas que los hombres, le agregan una dimensión pintoresca y animada, que resulta siempre en relatos más interesantes, escenas más crébles y proporciona un carácter más natural a la obra completas. Sin embargo, en p. 3 había escrito: «Como se verá, los personajes femeninos constituyen un elemento necesario y unificador de la novelax. 
Cervantes como «un verdadero caballero, que respetaba a la mujer" 6 .

El estudio del erotismo y de la presencia de la mujer en el Quijote se ha llevado a cabo, en efecto, desde diferentes perspectivas. Me propongo en las páginas que siguen analizar con detalle tales perspectivas, que han abierto un campo de estudio amplio y fructífero sobre nuestra novela más universal.

\section{EL DESEO COMO MÓVIL QUIJOTESCO}

Los trabajos del investigador francés René Girard han abierto un campo de estudio sobre el Quijote que ha sido seguido por diversos hispanistas. En efecto, en libros como Mesonge romantique et vérité romanesque o La violence et le sacré ${ }^{7}$, entre otros, desarrolla una teoría del deseo como móvil básico de las obras literarias, que no duda en aplicar al Quijote. Según Girard, todo relato presenta tres elementos fundamentales: un sujeto que desea, un objeto deseado y un mediador de este deseo. En la novela cervantina, el personaje principal también se mueve en función del deseo, pero no hacia objetos o personas que él mismo elige, sino hacia aquellos que le indica un tercero, el mediador. Don Quijote, pues, es la persona que desea; los héroes de caballería a quienes quiere imitar son los mediadores; y en las diferentes aventuras que se suceden en la obra, todas a imitación - parodia- de las de los libros de caballerías, aparecen los objetos deseados, que cambian sucesivamente. Esta estructura tripartita sustituye al esquema convencional de sujeto que deseaobjeto deseado.

De igual forma, en función de la proximidad mayor o menor entre sujeto que desea y mediador, las novelas pueden clasificarse en novelas de mediación interna (distancia muy corta), o externa (distancia mayor). Don Quijote podría incluirse en el primer tipo, pero no de manera radical, pues, tal como ha señalado Donatella Pini Moro, en la novela de Cervantes se produce una

- Ibidem, p. 8.

7 RENE GIRARD, Mesonge romantique et vérité romanesque. Paris, Grasset, 1961, traducción española por Guillerno Sucre bajo el título Mentira romántica y verdad novelesca. Caracas, Universidad Central de Venezuela, 1963; La violence et le sacré. Paris, Grasset, 1972. Vid. finalmente Des choses cachées depuis de la fondation du Monde. Parí, Grasset \& Fasquelle, 1978; y Le bouc émissaire. París, Grasset \& Fasquelle, 1982. 
«transformación y convulsión que la lleva a ser de externa a interna ${ }^{8}$.

Interesantes son, dentro de esta teoría de Girard, su análisis de la muerte de don Quijote, interpretada no sólo como la manera de concluir, sino, asimismo, como la reconciliación de aquél con el mundo; las posibles conexiones entre este personaje y el protagonista de la novela de Dostoyevski El eterno marido, el ascenso del caballero según esta teoría del deseo, etc. Girard estudia, no obstante, algunos episodios y capítulos del Quijote, pero no ofrece un análisis completo de la novela cervantina siguiendo su teoría del deseo.

Esta manera de estudiar el Quijote ha tenido, tal como indico al comienzo de este apartado, algunos continuadores. Quizás el principal de ellos sea Cesáreo Bandera, que ha dedicado un libro entero, Mimesis conflictiva ${ }^{9}$, a desarrollar las teorías de Girard y aplicarlas a episodios concretos del Quijote. El libro, desencadenador de una intensa polémica ${ }^{10}$, lleva prólogo del propio René Girard.

En su obra, Cesáreo Bandera profundiza en la caracterización de ese deseo de los personajes como búsqueda de un "otro inasiblex, cuyo nivel más elemental es el de la diferencia de sexo. En este contexto, resalta aquellos elementos que acaso pudieran haber interesado más a Cervantes:

* A Cervantes no sólo le interesa poner de manifiesto la presencia del mediador, o sea, la estructura triangular del deseo, sino asimismo y sobre todo revelar que ese mediador que absorbe por completo la atención del sujeto, que es venerado por éste, es de hecho un dios falso, un ídolo fabricado por el deseo. En otras palabras, a Cervantes le in-

- Donatella Pini Moro, «El Quijote y los dobles: sugerencias para una relectura de la novela cervantina*, Actas del 1 Coloquio Internacional de la Asociación de Cervantistas. Barcelona, Anthropos, 1990, p. 226.

- Cesareo Bandera, Mimesis conflictiva. Ficción literaria y violencia en Cervantes y Calderón. Madrid, Gredos, 1975. Vid. también su trabajo posterior «Deseo y creación literaria en el Quijote», MLN, 95, 1980, pp. 279-94.

10 En efecto, el libro de Bandera ha originado una interesante polémica que puede seguirse a través de las sucesivas réplicas y contrarréplicas que sostuvo en 1981, con RUth El Saffar ( $*$ On Beyond the Conflict*, Cervantes, I, 1-2 [1981], pp. 83-94, y «Response to Cesáreo Bandera», ibidem, pp. 108-10; BANDERA, *An Open Letter to Ruth El Saffar, ibidem, pp. 95-107); en 1982, con ARTHUR EFRON («Bearded Waiting Women, Lovely Female Piratemen: Sexual Boundary Shifts in Don Quixote», Cervantes, II, 2 [1982], pp. 155-64, y *On Some Central Issues in Quixote's Criticism: Society and the Sexual Bodyw, ibidem, pp. 171-83; BANDERA, *Healthy Bodies in Not-So-Healthy Minds», ibidem, pp. 165-70); y en 1985, con Robert Ter Horst (Bandera, *About 'Female' Art, 'Male Silence', and the Frivolous in General», Cervantes, V, 1 [1985], pp. 45-57; Ter Horst, "On the Importance of Being Earnest: A Reply to Cesáreo Bandera», ibidem, pp. 59-63). 
teresa sobre todo poner de manifiesto el proceso a través del cual el sujeto de deseo ficcionaliza la realidad, la convierte en literatura ${ }^{11}$.

Otro de los aspectos que cabe destacar en este trabajo de Bandera es la relación que establece entre don Quijote y Cardenio, personajes ambos movidos por ese deseo que necesita un mediador.

Louis Combet, también en la línea de los trabajos de Girard, desarrollándolos o, en ocasiones, apartándose de ellos, ha expuesto su idea de la existencia en toda la obra cervantina de un principio estructurante de raíz psicológica. Este principio, que presenta un carácter esencialmente masoquista, estaría en la base de las motivaciones de los personajes que aparecen no ya sólo en el Quijote, sino en toda la producción cervantina ${ }^{12}$.

Su libro, sin embargo, acaba convirtiéndose en un catálogo de los tipos de mujeres que se pueden encontrar en las obras de Cervantes (la mujer violenta, cruel, sádica, decidida, activa, inteligente,...); de los tipos de relaciones sexuales que sostienen presumiblemente los diferentes personajes (masoquismo, efebismo, comportamientos fetichistas y homosexuales); de los tipos de refugio que buscan los mismos personajes (locura, melancolía, escapismo, viajes, peregrinaciones, carnaval, mendicidad...); etc.

Asimismo, intenta utilizar algunos elementos de la biografía de Cervantes para que ayuden a explicar este principio psicoestructurante. Pero, bien por falta de datos concretos (como es sabido, períodos grandes de la vida de Cervantes están todavía por conocerse con detalle), bien por interpretaciones muy alejadas de los propios documentos, en mi opinión, el propósito de Combet se queda en eso, en un propósito.

Donatella Pini Moro se muestra también convencida de la validez de las ideas de Girard y ve en ellas la clave que

"permite aprehender el mecanismo de fondo quic da vida a los personajes cervantinos más variados y aparentemente heterogéneos y lejanos: un mecanismo que en cierto modo materializa y lleva a las últimas consecuencias la problemática teórica de la imitación ${ }^{13}$.

Con este fundamento teórico, propone una explicación del

1 BANDERA, Mimesis conflictiva, ob. cit., p. 148.

12 Vid. Cervantès ou les incertitudes du désir. Une approche psychoestructurale de l'oeuvre de Cervantes. Lyon, Presses Universitaires de la France, 1980. A este principio de carácter masoquista ya hacía referencia, siquiera de pasada, René Girard en el prólogo al libro de Cesáreo Bandera, ob, cit., p. 10.

13 Donatella Pini Moro, art. cit., p. 228. 
cambio de estructura que se produce en la novela a partir del capítulo 23 de la primera parte, cuando los personajes llegan a Sierra Morena. Alli empieza a introducirse una serie de dobles (Cardenio y Anselmo, por ejemplo) en los que se puede reproducir el esquema del deseo (sujeto que desea, objeto deseado y mediador) propuesto por Girard. De igual manera, la penitencia quijotesca se interpreta como el «ascenso en nombre del deseon de que habla Girard, y que Pini Moro cree fundamental para comprender el referido episodio ${ }^{14}$.

Edgar Paiewonsky Conde y Steven Hutchinson han abordado otros aspectos del deseo en el texto cervantino. El primero defiende que todo el Quijote está impregnado de la doctrina de Ficino del deseo erótico, lo cual, además, se puede hacer extensible al resto de sus obras ${ }^{15}$. En cambio, Steven Hutchinson, persuadido de que «Discourse about desires constitutes a central leitmotiv in all of Cervantes' novels ${ }^{16}$, propone, con elementos procedentes de los estudios de René Girard y, sobre todo, de una amplia gama de ejemplos extraídos de las propias obras cervantinas -incluidas las Semanas del Jardin, de tan dudosa autoría -, una caracterización del deseo tal como aparece en los textos de Cervantes. Según Hutchinson, el deseo supone, ante todo, movimiento, esto es, hace mover a los personajes y motivarlos.

\section{LA PERSPECTIVA FILOLOGICA}

Otros trabajos ha abordado, desde una perspectiva filológica, aspectos variados de la presencia y función del erotismo en el Quijote.

Teresa Aveleyra, por ejemplo, en uno de los primeros estudios sobre la cuestión, caracteriza a don Quijote como un tímido erótico, esto es, un personaje que, pese a su timidez, posee una atracción muy grande hacia la mujer. Como consecuencia

* La represión la transforma en un erotismo hiperestético que funciona en dos maneras principales: sublimando al objeto erótico hasta espirirualizarlo por completo; o dejándolo como tal objeto erótico:

\footnotetext{
14 Ibidem, pp. 228-9.

15 Es la doctrina del appetitus naturalis, según la cual «todas las cosas que existen siempre desean algo». Vid. Edgar PaIEwONSKY CONDE, *Cervantes y la teoría renacentista del deseo», ACer, XXIII, 1985, p. 77.

16 Steven Hutchinson, «Desire Movilized in Cervantes' Novels», JHP, 14, 1990. p. 159.
} 
deseable pero inaccesible, situado detrás de las barreras psíquicas creadas por la mente misma del caballero ${ }^{17}$.

Ruth S. Lamb, que sigue muy de cerca a Joaquín Casalduero, ha querido ver en Marcela una representante de la mujer renacentista, mientras que en Dorotea una representante de la mujer barroca, «inscrita en la actualidad y en un medio socialurbano» ${ }^{18}$. Michèle Gendreau-Massaloux, en cambio, analiza tres episodios quijotescos en los que aparecen locos de amor: la historia de Cardenio, la penitencia de don Quijote imitando a Amadís y la locura de Roldán y el falso suicidio de Basilio. Los tres episodios "se insertan en el intento irónico de su creador", es decir, están al servicio del propósito paródico de Cervantes en la novela ${ }^{19}$.

Por su parte, Salvador J. Fajardo estudia la manera sutil mediante la cual Cervantes presenta a Dorotea. Cervantes introduce al lector en una atmósfera erótica, que se va intensificando conforme el capítulo avanza, hasta que por fin se descubre que el personaje en cuestión es una mujer:

«The emergence of Dorotea is orchestrated with great care. In the following remarks I consider the principal stages of this event with special attention to the narrative and descriptive strategies that set it forth and to the impact of the episode's underlying eroticism ${ }^{20}$.

Agustín Redondo ha recordado uno de los motivos fundamentales de la aparición - directa o indirecta - del erotismo en esta obra cervantina: el Quijote es, en primer lugar, una parodia de los libros de caballerías, obras donde aparecen muchos pasajes, situaciones, motivos y episodios de carácter erótico. En consecuencia, es un elemento que, lógicamente, ha de aparecer por fuerza en esta novela de Cervantes. Y el erotismo, en opinión del profesor Redondo, se muestra de dos modos: uno "primero, bur-

17 Teresa Aveleyra, «El erotismo de don Quijote», NRFH, XXVI, 1977, p. 472.

18 RUTH S. LAMB, «Las mujeres del Quijote: contraste entre la mujer renacentista y la mujer barroca», Manuel Criado de Val (ed.), Cervantes, su obra y su mundo. Actas del I Congreso Internacional sobre Cervantes. Madrid, EDI-6, 1981, p. 769. De Joaquin Casalduero, vid. su conocido libro Sentido y forma del "Quijote". Madrid, Insula, 1975, 4." ed.; y el artículo "La sensualidad del Renacimiento y la sexualidad del Barroco. Por qué Cervantes rechaza la pastoril y no acepta la picaresca*, Edad de Oro, III, 1984, pp. 29-31.

19 Michele Gendreau-Massaloux, aLos locos de amor en el Quijote. Psicopatología y creación cervantina», Manuel Criado de Val (ed.), Cervantes, su obra y su mundo, ob. cit., p. 687.

${ }^{20}$ Salvador J. Fajardo, «Unveilling Dorotea or the Reader as Voyeur», Cervantes, IV, 2, 1984, pp. 89-90. 
lesco y ampliamente dominante, y el segundo, natural y agradable, pero ocasional" ${ }^{21}$. Se examinan ambos, pero s6lo con ejemplos extraídos del Quijote de 1605. El primero de ellos se puede observar en las diversas escenas en las ventas, en el episodio de Rocinante con las yeguas $(I, 15)$, etc. $Y$, junto a este erotismo burlesco,

*hay en el Quijote, fuera ya de este universo parodico, un momento de intensa y auténtica fruición sensual, que corresponde con la aparición de Dorotea (I, 28). El marco es el de la sierra, o sea, el de esa Naturaleza que permite liberarse de las coerciones impuestas por la vida socials 22 .

Monique Joly ha estudiado en dos ocasiones aspectos distintos de la presencia de la mujer en la novela cervantina. En el primero de estos trabajos muestra cómo los personajes femeninos son caracterizados y presentados en función de la verosimilitud y decoro de la obra:

- Lo que me interesa es examinar un aspecto poco atendido de la invención cervantina, poniendo de realce cómo el eros caballeresco, al convertirse en motor de la conducta de don Quijote frente a cualquier 'dama', repercute por lo riguroso de las exigencias cervantinas en materia de decoro, en la caracterización de los personajes femeninos de la novela, y singularmente en su caracterización verbal ${ }^{23}$.

Con estas premisas analiza agudamente la aparición en la obra de algunos personajes femeninos: la esposa de don Diego de Miranda (II, 18), las mujeres que aparecen en casa de don Antonio Moreno (II, 62), el personaje de Altisidora (II, 69), etc.

En artículo posterior cambia de perspectiva y analiza muy detalladamente un aspecto de la caracterización de Sancho Panza, en concreto, el que se relaciona con su posible capacidad sexual, limitada sólo al terreno verbal.

En efecto, tomando como punto de partida las palabras, llenas de connotaciones eróticas, proferidas por este personaje en la casa de don Diego Moreno, tras la exhibición de baile de Don Quijote $(* \mathrm{Si}$ hubiérades de zapatear, yo supliera vuestra falta, que zapateo como un girifalte; pero en lo del danzar, no doy pun-

21 Agustin Redondo, «Las dos caras del erotismo en la primera parte del Quijotew, Edad de Oro, IX, 1990, p. 253.

22 Ibidem, p. 264. Sobre la aparición de Dorotea en la novela ha de tenerse en cuenta también el trabajo de Salvador J. Fajardo citado anteriormente.

${ }^{23}$ Monique Joly, kla sexualidad en el Quijote», Edad de Oro, IX, 1990, pp. 137-8. 
tada ${ }^{24}$ ), la profesora Joly expone su hipótesis, según la cual sancho es incapaz sexualmente. Tal rasgo está ya presente, al menos, desde el capítulo 28 de la primera parte del Quijote:

«El salto que me propongo dar ahora es mayor todavía, en la medida en que voy a sugerir que el modelo inicial de estas expresivas alusiones de Sancho a su añoranza de no haber podido mostrar que es más gallo que su señor, que por dos veces encontramos en los quince últimos capítulos de la Segunda Parte, está en realidad presente en la obra desde que por primera vez aparece en ella una situación parecida a las anteriormente descritas, a saber, desde el momento en que la trayectoria de Dorotea se cruza con la de Sancho y Don Quijote» ${ }^{25}$.

Alfredo Baras Escolá profundiza en las posibles connotaciones eróticas del nombre Quijote partiendo de la siguiente premisa: "Leyendo con atención a Cervantes, se descubre que el pleno valor alusivo de su obra sólo es posible apreciarlo gracias al equívoco usado como recurso" ${ }^{26}$. Los elementos del nombre que examina son sus diversas variantes (Quexada, Quijada, Quesada, etc.) y los valores posibles del sufijo -ote ${ }^{27}$. Finalmente, Jacques Joset ensaya una posible justificación de la casi total ausencia de la familia de don Quijote. Ésta se explica, tal como Juan Bautista Avalle-Arce y Antonio Rey Hazas han expuesto acertadamente, en función de la propia teoría literaria cervantina, que tiene en la libertad su principio fundamental. Joset concluye: "Cervantes ubica a don Quijote en un espacio de libertad literaria casi sin límites [...] La ocultación de la familia pertenece, pues, al proyecto cervantino de contar la vida de un individuo» ${ }^{28}$.

24 Miguel de Cervantes, Don Quijote de la Mancha. Edición crítica y comentario de Vicente Gaos. Madrid, Gredos, 1987, vol. II, p. 880. La cita corresponde al capítulo sesenta y dos de la segunda parte.

${ }^{25}$ Monioue Joly, «Erotismo y marginación social en la novela cervantinas, art cit., p. 11. Vid. también la p. 13: «[las] esporádicas alusiones de Sancho a su propio vigor sexual que encontramos en varios lugares de la obra, y que yo interpreto como otras tantas exhibiciones fálicas, se entiende que restringidas al terreno de la expresión verbal». Mauricio Molho ha puesto de relieve también aspectos diversos de la caracterización de Sancho que permiten al investigador francés hablar de la feminidad de este personaje. Vid. MaUricio MolHo, «Doña Sancho (Quijote, II, 60)», Homenaje a José Manuel Blecua. Madrid, Gredos, 1983, pp. 443-8.

26 Alfredo Baras Escolá, «Una lectura erótica del Quijote», Cervantes, XII, 2, fall 1992 , p. 79.

27 Sobre estas cuestiones sigue siendo fundamental el libro de ÁNGEL RosenBLAT, La lengua del "Quijote". Madrid, Gredos, 1971.

28 JACOUES JOSET, «De la familia de don Quijote y de la sobrina de éste o 'Familles, je vous hais!'», Actas del II Coloquio Intemacional de la Asociación de Cervantistas. Barcelona, Anthropos, 1991, p. 132. Vid. asimismo JuAn Bautista 


\section{LA INTERPRETACION PSICOANALITICA}

Otra línea de investigación propone estudiar los personajes del Quijote como si de personas reales se tratara. Éstos, por tanto, son susceptibles de analizarse a la luz de la psicología moderna, en algunas de sus posibles vertientes: psicoanálisis freudiano, psicología americana del ego, teorías de Jung, etc. Carroll B. Johnson, quizás el más destacado exponente de esta manera de analizar la obra cervantina, así lo proclama:

«...this work's basic aesthetic criterion is that of verosimilitude, circumstantial and especially psychic. It means that the characters it portrays think and act as real people think and act, that their behavior, like ours, is overdetermined -that is, it responds to both conscious and unconscious motivation simultaneously - that they are sensitive, as we are, to life's pressures and mount unconscious defenses to deal with them, and that their 'characters' are in part the result of the particular kinds of defenses to which they typically have recourse ${ }^{29}$.

Este tipo de estudio sobre el Quijote tiene un lejano precedente en la conocida Guía del lector del "Quijote", de Salvador de Madariaga, donde se efectuaban consideraciones de carácter psicológico sobre los personajes de la novela cervantina ${ }^{30}$. Otros antecedentes de esta clase de análisis pueden encontrarse también en algunas de las obras de René Girard y en el conocido libro de Marthe Robert, L'ancien et le nouveau. De "Don Quichotte" a Franz $\mathrm{Kafka}^{31}$, en el que había expuesto su teoría de que hay dos clases de novelas: las que pertenencen al tipo del

Avalle-Arce, «Tres comienzos de novela. (Cervantes y la tradición literaria. Segunda perspectiva)», capitulo tercero de su libro Nuevos deslindes cervantinos. Barcelona, Ariel, 1975, pp. 213-43; y Antonio Rey Hazas, «Cervantes, el Quijote y una poética de la libertad , Actas del I Coloquio Internacional de la Asociación de Cervantistas. Barcelona, Anthropos, 1990, pp. 369-80.

${ }_{29}$ Carroll B. Johnson, Madness and Lust. A Psychoanalitical Approach to "Don Quixote". Berkeley, University of California Press, 1983, p. 197.

${ }^{30}$ Este libro data de 1923 y lleva como significativo subtítulo «Ensayo psicológico sobre el Quijote (manejo la séptima edición, Buenos Aires, Editorial Sudamericana, 1972). La traducción al inglés lleva por título "Don Quixote": An Introductory Essay in Psychology. Oxford, Clarendon Press, 1961, 2.: ed, revisada. Precisamente, ha sido en los países anglosajones donde este tipo de análisis ha tenido (y sigue teniendo) mayor aceptación. Pionero en este tipo de trabajos fue SAntiago Ramón y Cajal con aLa psicología de Don Quijote de la Mancha y el quijotismox, VV. AA. Discursos lefdos en la sesión solemne que el Colegio de Médicos de la provincia de Madrid dedica a Miguel de Cervantes. Madrid, Imprenta de J. A. Garcia, 1905, pp. 37-59. Vid. finalmente José Manuel Bailón Blancas, Historia clinica del caballero don Quijote. Madrid, Imp. Publicidad Impresora Madrileffa, 1989.

${ }^{31}$ Paris, Grasset, 1962. 
niño expósito, y las que pertenecen al tipo del niño bastardo. El Quijote, para Robert, ha de ser incluido en el primero de ellos, pues el protagonista principal deambula por un ámbito geográfico anacrónico con el ambiente cultural (el de las novelas de caballerías) en el que se inserta el personaje ${ }^{32}$.

En esta línea de análisis, John G. Weiger ha estudiado, utilizando las teorías sobre la personalidad de Carl G. Jung, el proceso de individuación que, a su parecer, tiene lugar en el personaje de don Quijote, hasta que éste se hace consciente de sí mismo. Se trata, en definitiva, de desarrollar el conocido tema cervantino «cada uno es hijo de sus obras", analizado aquí con elementos procedentes de ciertas ramas de la psicología moderna:

*That process of sustaining one's existence, without any necessary recourse to the political connotations of the word 'individualism', is the need of humanity to find a niche in one's lifetime which carries meaning for the individual, 33 .

Esta individuación se produce sobre todo a través de la creación de una amada ideal, inalcanzable físicamente:

-The protagonist simply becomes aware of the emptiness and futility of his life, a life of failure in any arena, but particularly in those connected with virility (sexual and warrior prowess), and, fearing it is too late to attempt any real sexual conquest, he invents an ideal lover

32 Sobre estos autores y, en general, la corriente psicoanalitica aplicada a las obras literarias con referencias concretas al Quijote, puede verse el breve artículo de José Romera CASTILlo, «Don Quijote como alter ego de Cervantes», Manuel Criado de Val (ed.), Cervantes, su obra y su mundo, ob. cit., pp. 493-9. Vid. asimismo ANne Clancier, Psicoanálisis. Literatura. Critica. Madrid, Cátedra, 1976, pp. 220-2; Elizabeth Wright, Psychoanalytic Criticism: Theory in Practise. Londres y Nueva York, Methuen, 1984; y MARtA Grazia ProfeTI, *Literatura y estudio biográfico. Psicoanálisis y literatura», José María Díez Borque (coord.), Métodos de estudio de la obra literaria. Madrid, Taurus, 1989, reimpresión, pp. 313-52. Por otra parte, LeON Grimberg y Juan Francisco Rodriguez ( \&La influencia de Cervantes sobre el futuro creador del psicoanálisis», $A C e r$, XXVXXVI, 1987-1988, pp. 157-74) y E. C. RILEY («Cervantes y Freud», Insula, 538, octubre, 1991, pp. 34-5; y «Cervantes, Freud, and Psychoanalytic Narrative Theory», Modern Language Review, 88, 1, January, 1993, pp. 1-14) han llamado la atención sobre la posible importancia e influjo de la lectura de algunas obras cervantinas por Freud en la elaboración de su teoría del psicoanálisis. Finalmente, el estudio de otras obras cervantinas desde la perspectiva psicoanalítica cuenta también con trabajos diversos. Vid. JoHN E. Gedo y ERNEST S. Wolf, «Freud's Novelas ejemplares», Psychological Issues, 34-35, 1976, pp. 87-88; y S. B. VRANICH, «Sigmund Freud and 'The Case History of Berganza': Freud's Psychoanalytic Beginnings», Psychoanalytic Review, 63, 1976, pp. 73-82.

33 JoHN G. WEIGER, The Individuated Self: Cervantes and the Emergence of the Individual. Athens, Ohio University Press, 1979, p. 28. 
who, as his thorough reading of the novels of chivalry has informed him, need not be physically attainable. What is more, having declared such an ideal courtly love for Dulcinea, he is now free to avoid any other real love entanglements with the convenient pretext that any amorous desires he may feel must be limited to Dulcineas ${ }^{34}$.

Como he indicado antes, ha sido Carroll B. Johnson, profesor de la Universidad de California, el que de manera más destacada ha efectuado este tipo de acercamiento al Quijote, en trabajos en los que utiliza no sólo las teorías de Freud, sino estudios más recientes de la psicología americana del ego, en particular aquellos que tratan de la crisis de madurez en los hombres. Con ellos ha conformado un cuerpo de exégesis sobre el Quijote serio, bien sustentado, pero muy discutido y polémico.

En el principal de estos trabajos, su libro Madness and Lust ${ }^{35}$, considera a Don Quijote como si de una persona real de nuestra época se tratara: un hombre en la crisis de los cincuenta anos. Johnson, pues, caracteriza esta crisis y la aplica a nuestro personaje, el cual, de acuerdo con la hipótesis sugerida por el crítico, se lanza a leer libros de caballerías debido a que su vida es triste y aburrida y necesita escapar hacia un mundo de sexo y violencia para permanecer sano. Don Quijote no se vuelve loco por los libros, lee los libros en un esfuerzo por no volverse loco; huye de su casa no en busca de Aldonza Lorenzo, sino por huir de su sobrina, de quien está enamorado en secreto. Don Quijote se mueve, pues, entre la atracción hacia las mujeres y el terror que al mismo tiempo le producen. A la luz de estas premisas se analizan los diversos encuentros de este personaje con personajes femeninos: Marcela, la hija del ventero, Dorotea, Doña Rodríguez, y Altisidora, quien, según Johnson, resuelve el problema a Don Quijote.

En el libro citado y en un trabajo posterior, aplica estas consideraciones a otros personajes de la novela, llegando a la conclusión de que es la sexualidad de los personajes la que alos define como individuales y los hace interesantes para nosotros" ${ }^{36}$. En ella -en la sexualidad- residiría para este crítico uno de los grandes alicientes de la novela:

«Lo que Cervantes parece estar comunicando es simplemente lo frágil, lo problemática que son la identidad sexual y la expresión de la sexualidad. Cervantes dramatiza las dificultades de ser hombre y de ser

34 Ibidem, p. 70.

${ }^{35}$ Obra que ya he citado con anterioridad.

36 Carroll B. Johnson, "La sexualidad en el Quijote", Edad de Oro, IX, 1990, p. 136. 
mujer. Y porque nunca deja de ser Cervantes, atrae al lector hacia la problemática y le mete dentro. A medida que uno lee, su propia identidad sexual entra en juego, se encuentra involucrada y puesta en tela de juicio. Se trata sólo en parte, por ejemplo, de cómo vive Marcela las dificultades de su identidad sexual; también implica cuestiones de quién lee a Marcela, a Altisidora, a Don Quijote y a Anselmo* ${ }^{37}$.

No es necesario resaltar, lo arriesgado de estas hipótesis, que han dado lugar a opiniones encontradas: es posible señalar reparos tanto de carácter general (consideración de los personajes como si fueran personas de carne y hueso; validez de la aplicación de criterios o métodos de análisis de nuestra época a momentos históricos en que se desconocían, etc.); como de carácter más concreto (utilizar determinados elementos que pueden favorecer la referida interpretación dejando de lado otros que posiblemente la puedan anular o contrarrestar; en 1600 una persona de cincuenta años no se encuentra como ahora en la mitad de la vida, sino que es verdaderamente un anciano; etc.). Es de justicia también reiterar la seriedad y rigor demostrados por el profesor Johnson, quien ya advertía sobre la posible acogida de este tipo de trabajos: «I do not expect this book to be received with enthusiasm by most of my fellow Hispanists. I ask only that it be received with an open mind and taken as seriously as I have attempted to take Cervantes and Don Quixote" ${ }^{38}$.

En esta línea de interpretación psicoanalista, John T. Cull ha examinado algunos símbolos que pudieran ser indicadores de la impotencia sexual de don Quijote: el brazo inhábil y la lanza rota. Relaciona asimismo estos símbolos con la lectura cómica del Quijote propuesta por una parte de la crítica anglosajona:

*Any suggestion of Don Quixote's impotence is not to be taken too seriously nor used as ammunition to mount an argument on his creator's sexuality. It is all part of the elaborate trick that Cervantes plays on his protagonist by making him a hilarious parody of the Knights of Chivalry: ${ }^{39}$.

\section{EL «QUIJOTE» Y EL FEMINISMO}

Partiendo igualmente de la premisa de considerar a los protagonistas de la novela cervantina como si se tratase de perso-

37 Ibidem.

38 Carroll B. Johnson, Madness and Lust, ob. cit., p. 9.

39 JoHN T. CULL, *The 'Knight of the Broken Lance' and His 'Trusty Steed': On Don Quixote and Rocinante», Cervantes, X, 2, fall, 1990, p. 50. 
nas reales, Ruth El Saffar ha elaborado numerosos trabajos sobre el Quijote y otras obras cervantinas, pero ampliando la perspectiva.

En efecto, la hispanista norteamericana combina en sus trabajos ideas que proceden de estudios sobre la presencia de la mujer y el erotismo de Cervantes - René Girard, psicoanálisis en sus distintas vertientes (Freud, Jung, Lacan), psicología americana del ego, crítica feminista de última hora...-, con otros de corte más clásico (Northrop Frye, Edward C. Riley, Alban K. Forcione) que abogan por la aplicación de la dicotomía romance / novela a la obra cervantina. Forma con ellos, de manera similar a como sucedía con los de Carroll B. Johnson, un cuerpo de exégesis de la novela cervantina serio, pero discutible por su excesivo alejamiento a veces del texto del Quijote.

En un artículo suyo aparecido en 1983, anticipo de algunos aspectos desarrollados luego en su libro Beyond Fiction, El Saffar estudia elementos que se asocian inconscientemente con lo femenino: el agua, la cueva, la noche, etc. El agua se asocia de forma constante a la presencia de las mujeres, como símbolo de pureza, frescor, belleza, vida, fertilidad, etc. El estudio de la manera en que aparecen tratados estos elementos en las dos partes del Quijote conduce a El Saffar a elaborar unas conclusiones de carácter general sobre la presencia y función de lo femenino en el Quijote:

*Along with water, caves, moon, night animals, etc., we find in Part II a great increase in the number of women who are active, married and yet independent [...] In Don Quixote Part I the cluster of elements associated with the masculine - the sun, the hard, dry earth, travel, adventure, homelessness - underscore the alienation and alienating effect of the female characters with it. In Don Quixote Part II an underground rumbling, like an earthquake, breaks up the harsh dry terrain of the hero's travels, introducing into the narrative sign of darkness, liquidity, and enclosure. The eruption of such feminineassociated forces comes against the grain of the will, however, and emerges in fierce, frightening aspect. ${ }^{40}$.

Al año siguiente, en 1984, Ruth El Saffar publica un extenso libro en el que pretende llevar a cabo una nueva interpretación general de toda la obra cervantina, ahora desde una perspectiva diferente a la empleada unos años antes ${ }^{41}$. En efecto, en Novel

40 RUth El SAFFar, *Fiction and Androgyne in the Works of Cervantes», Cervantes, III, 1, 1983, pp. 47 y 49.

${ }^{41}$ RUTH EL SAFPAR, Beyond Fiction. The Recovery of Feminine in the Novels of Cervantes. Berkeley, University of California Press, 1984. 
to Romance ${ }^{42}$ había postulado una evolución consciente en Cervantes desde la forma narrativa que la crítica anglosajona denomina novela hacia el romance. Esta hipótesis fue en su momento rechazada, entre otros motivos, porque el primer libro de Cervantes, La Galatea, es, evidentemente, un romance en el sentido inglés del término ${ }^{43}$.

En Beyond the Fiction Ruth El Saffar acude a otros parámetros para considerar las cuatro novelas largas de Cervantes como un todo que evoluciona desde la primera de ellas, hasta que alcanza su plenitud en el Persiles.

La obra cervantina se considera ahora como un proceso de depuración que comienza en La Galatea y culmina en el Persiles. A lo largo de este proceso, Cervantes ha ido puliendo sucesivamente aquellos elementos (estilo, narración, descuidos, etc.) que consideraba desacertados, a la par que se produce una evolución progresiva en el tratamiento de la mujer. Conforme se pasa de una obra a otra, los personajes masculinos modifican paulatinamente su concepto de la mujer, hasta que, en el Persiles, llegan a aceptarlas por completo, tal y como ellas son. Esta obra póstuma de Cervantes se convierte así, en el parecer de la profesora El Saffar, en la culminación de este proceso y, por tanto, de toda la carrera literaria cervantina. Los pasos de dicha evolución han sido resumidos así por la propia autora:

"In La Galatea, Elicio explored what was closest to him -the male world of friends and rivals. The love one remained but a hazy image in that first work, poorly differentiated and inaccesible. Don Quixote Part I carried the development a step further by widening the range of male characters and deepening the hero's exploration of conflict and rivalry. The woman, meanwhile, emerged from her abstract perfection to offer a negative image to balance the overly positive one of the pastoral and chivalric traditions. Don Quixote Part II showed the hero probing the unknown, while his principal alter ego, Sancho, freed himself to undergo a process of transformation paralell to Don Quixote's, breaking down at the same time the relation of master to servant that prevailed in Part I. In the Persiles the hero was totally inmersed in the alien, masterful in his dealings with his same sex rivals, and attoned to the point of symbolic identity with the loved one. The process showed movement from periphery to center, allowing for both increasing unity ${ }^{44}$.

Ahora bien, resulta dificil considerar el Persiles como culmi-

${ }^{42}$ RUth EL SAffar, Novel to Romance. A Study of Cervantes' "Novelas ejemplares". Baltimore, Johns Hopkins University Press, 1974.

${ }^{43}$ Vid. el prólogo de Florencio Sevilla Arroyo y Antonio Rey hazas a su edición de las Novelas ejemplares, Madrid, Espasa-Calpe, 1991, p. 16.

44 Ruth El Safrar, Beyond Fiction, ob. cit., pp. 170-1. 
nación de la carrera literaria de Cervantes - pese a que el mismo autor habla de él como el mejor libro que había escrito ade los de entretenimiento"-. Así sucede, en efecto, porque, como ha puesto de relieve la crítica más autorizada al respecto, se trata de una obra compuesta en diversos momentos de la vida de su autor $\mathbf{y}$, por tanto, con elementos que pueden encontrarse en el resto de sus obras.

De igual forma, creo que para buscar un nexo común a toda la labor literaria cervantina -si es que esto es posible- habría que dirigirse a terrenos que a su autor sí le preocupaban realmente (iy de qué manera!), como, por ejemplo, el de la teoría literaria; pero no en motivaciones del subconsciente cuya aplicación a las obras literarias, el Quijote en particular, no siempre es provechosa.

El asedio de Ruth El Saffar al Quijote desde estas posiciones de teoría feminista ha dado ocasión a tres trabajos más en los que aborda otros aspectos de la universal novela. En el de 1988 acude a estudios de Freud donde defiende que en la novela la mujer significa sobre todo carencia. Asimismo, reitera sus ideas sobre el diferente tratamiento de las mujeres en las dos partes del Quijote:

*What stands out in Part I and then emerges in Part II as a dominant theme, however, is the real power that belongs to the 'defendseless' women over whom the men ostensibly struggle. ${ }^{45}$.

Esto proporciona a la novela cervantina un aspecto subversivo que la hispanista norteamericana desarrolla en el artículo titulado "Voces marginales y visión del ser cervantino" ${ }^{46}$. Allí resalta igualmente el aspecto "feminista» de las obras cervantinas:

«Para comprender el aspecto 'feminista' de Cervantes, hay que ver sus obras bajo la luz de la construcción del ser moderno que se iba creando a fuerza de expulsión y represión a lo largo del siglo xvi. La atención que presta Cervantes a los que quedan fuera del discurso dominante es una con su fidelidad a un ideal prepatriarcal, ideal articulado por Don Quijote en su discurso sobre la Edad de Oron ${ }^{47}$.

Finalmente, en el homenaje que diversos hispanistas tributaron en 1989 a Elías L. Rivers, Ruth El Saffar desarrolla la posible presencia en la locura de don Quijote de lo que el crítico

4 Ruth EL SAFrar, «In Praise of What is Left Unsaid: Thoughts on Women and Lack in Don Quijote», MLN, 103, 2, 1988, p. 217.

to Anthropos, 98-99, 1989, pp. 59-63.

47 Ibidem, p. 62. 
norteamericano Erich Neuman ha denominado «the archetypal hero son of the Virgin Mother" ${ }^{48}$. Postula también la pertinencia de la aplicación al Quijote de los cultos a la Gran Madre que críticos psicoanalistas han relacionado con los mitos de Perséfone y Deméter. Todos ellos son símbolo de una sociedad matriarcal.

En esta misma línea de análisis, Yvonne Jehenson, por su parte, analiza el episodio de Marcela con ideas procedentes del feminismo francés y anglo-americano. El episodio se concibe ahora como "a subversive knowledge of the class and gender relations of the society whitin which the work has been produced and with those values it is often at odds" ${ }^{49}$. Finalmente, Marcela acaba convirtiéndose, a los ojos de Jehenson, en la mujer liberada que Cervantes podría tener en mente:

"In this episode [...] Cervantes has dared to slip something by at odds with tradition [...] imagining the woman who would hold out against oppression and constitute herself as a superb, equal, hence 'impossible' subject, untenable in a real social framework. Such a woman Cervantes imagined in Marcela ${ }^{50}$.

Los trabajos aquí estudiados revelan perspectivas y métodos de análisis muy diversos, próximos, unos, al texto cervantino; otros, en cambio, alejados, excesivamente alejados a veces, del Quijote. No han supuesto un avance sustancial para una mejor comprensión de la novela cervantina, pero muestran a las claras su singularidad y capacidad para ser estudiada e interpretada desde los prismas más variados. Y Don Quijote se yergue, por encima de todos ellos, manteniendo vivo su interés, su radical modernidad todavía hoy, casi cuatrocientos años después de su publicación.

\section{JOSE MONTERO REgUeRA}

48 Vid. RUth El SAFrar, \&Sex and the Single Hidalgo: Reflections on Eros in Don Quixote», Ruth El Saffar y Bruno Mario Damiani (eds.), Studies in Honor of Elias Rivers. Maryland, Scripta Humanistica, 1989, p. 85.

49 Yvonne Jehenson, «The Pastoral Episode in Cervantes' Don Quijote; Marcela Once Again*, Cervantes, X, 2, 1990, p. 17.

so Ibidem, p. 31.

Una vez entregadas estas páginas para su publicación he tenido noticia de dos importantes trabajos, que no he podido incorporar en el cuerpo de este artículo, y que introduzco ahora en esta nota final. Me refiero al estudio de E. C. RILEY, 'Cipion' Writes to 'Berganza' in the Freudian Academia Española», Cervantes, XIV, 1, Spring, 1994, pp. 3-18, en el que el autor continúa otro anterior suyo al que me refiero en nota $32 ; \mathrm{y}$ al libro Quixotic Desire. Psychoanalytic Perspectives on Cervantes, editado por RUTH ANTHONY El SAFfar y Diana de ARMas Wnson (Ithaca: Cornell University Press, 1993). Se trata de una interesante compilación, nueva muestra de la presencia y significación que este tipo de trabajos tiene en el hispanismo anglosajon. 


\section{BIBLIOGRAFIA CONSULTADA}

ALLEN, J. J., «El desarrollo de Dulcinea y la evolución de don Quijote», NRFH, XXXVIII, 2, 1990, 849-56.

Andrist, Debra D., *Male Versus Female Friendship in Don Quijote*, Cervantes, III, 2, fall, 1983, 149-59.

Avalle-Arce, Juan Bautista, *Tres comienzos de novela. (Cervantes y la tradición literaria. Segunda perspectiva)», capítulo tercero de su libro Nuevos deslindes cervantinos. Barcelona, Ariel, 1975, 213-43.

Aveleyra, TeresA, «El erotismo de Don Quijote», NRFH, XXVI, 1977, 468-79.

Bailón Blancas, José Manuel, Historia clinica del caballero don Quijote. Madrid, Imp. Publicidad Impresora Madrileña, 1989.

BANDERA, Cesareo, Mimesis conflictiva. Ficción literaria y violencia en Cervantes y Calderón. Madrid, Gredos, 1975.

-, «Deseo y creación literaria en el Quijote», MLN, 95, 1980, 279-94.

-. An Open Letter to Ruth El Saffar», Cervantes, I, 1-2, 1981, 95-107.

- . Healthy Bodies in Not-So-Healthy Minds", Cervantes, II, 2, 1982, 165-70.

-, «About 'Female' Art, 'Male Silence', and the Frivolous in General», Cervantes, V, 1, 1985, 45-57.

Baras EscolA, Alfredo, *Una lectura erótica del Quijote», Cervantes, XII, 2, 1992. 79-89.

Casalduero, Jonoutn, Sentido y forma del "Quijote". Madrid, Insula, 1975, 4." ed.

- . La sensualidad del Renacimiento y la sexualidad del Barroco. Por qué Cervantes rechaza la pastoril y no acepta la picaresca», Edad de Oro, III, 1984, 29-31.

Clancier, Anne, Psicoanálisis. Literatura. Critica. Madrid, Cátedra, 1976.

Сомвет, Lours, Cervantès ou les incertitudes du désir. Une approche psychoestructurale de l'oeuvre de Cervantès. Lyon, Presses Universitaires de la France, 1980.

Cull, JoHn T., *The 'Knight of the Broken Lance' and His 'Trusty Steed': On Don Quixote and Rocinante*, Cervantes, X, 2, 1990, 37-51.

CRUz, ANNe J., «Los estudios feministas en la literatura del Siglo de Oro», Manuel García Martín (ed.), Estado actual de los estudios sobre el Siglo de Oro. Salamanca, Ediciones Universidad de Salamanca, 1993, vol. I, 255-9.

Efron, ArthUR, «Bearded Waiting Women, Lovely Female Piratemen: Sexual Boundary Shifts in Don Quixote, Part II», Cervantes, II, 2, 1982, 155-64.

-, «On Some Central Issues in Quixote Criticism: Society and the Sexual Body», Cervantes, II, 2, 1982, 171-83.

El Saffar, Ruth S., Novel to Romance. A Study of Cervantes' "Novelas ejemplares". Baltimore, Johns Hopkins University Press, 1974.

—, *Tres imágenes claves de lo femenino en el Persiles *, RCEH, III, 1979, 135-60.

-, *On Beyond the Conflict*, Cervantes, I, 1-2, 1981, 83-94.

-. «Response to Cesáreo Banderan, Cervantes, I, 1-2, 1981, 108-10.

- . Fiction and Androgyne in the Works of Cervantes», Cervantes, III, 1, 1983, 35-49.

- Beyond Fiction. The Recovery of the Feminine in the Novels of Cervantes. Berkeley, University of California Press, 1984.

-, eIn Praise of What is Left Unsaid. Thoughts on Women and Lack in Don Quijote», MLN, 103, 2, march, 1988, 205-22.

-. «Sex and the Single Hidalgo: Reflections on Eros in Don Quixote», Ruth El Saffar y Bruno M. Damiani (eds.), Studies in Honor of Elfas Rivers. Potomac, Maryland, Scripta Humanistica, 1989, 76-93. 
-, "Voces marginales y la visión del ser cervantinon, Anthropos, 98-99, 1989. 59-63.

EsPinA, ConchA, "Mujeres del Quijote», Obras completas de Concha Espina. Madrid, Ediciones Fax, 1955, 2.* ed., vol. II, 826-52.

Fajardo, Salvador J., «Unveilling Dorotea or the Reader as Voyeur*, Cervantes, IV, 2, 1984, 84-108.

Gedo, John E. y Wolf, ERnest S., «Freud's Novelas ejemplares», Psychological Issues, 34-35, 1976, 87-88.

Gendreau-Massaloux, Michele, «Los locos de amor en el Quijote. Psicopatología y creación cervantinax, Manuel Criado de Val (ed.), Cervantes, su obra y su mundo. Actas del I Congreso Internacional sobre Cervantes. Madrid, EDI-6, $1981,687-97$.

GIRARD, RENE, Mesonge romantique et vérité romanesque. París, Grasset, 1961. Traducción española por Guillermo Sucre bajo el título Mentira romántica y verdad novelesca. Caracas, Universidad Central de Venezuela, 1963.

- La violence et le sacré. París, Grasset, 1972.

- Des choses cachées depuis de la fondation du Monde. París, Grasset \& Fasquelle, 1978.

-, Le bouc émissaire. París, Grasset \& Fasquelle, 1982.

Grimberg, León y Rodriguez, JuAN Francisco, "La influencia de Cervantes sobre el futuro creador del psicoanálisis», ACer, XXV-XXVI, 1987-1988, 157-76.

Hathaway, Robert L., claudia Jerónima (DQ, II, 60)», NRFH, XXXVI, 1988. 319-32.

Hutchinson, Steven, «Desire Mobilized in Cervantes' Novels», JHP, 14, 1990, 159 74.

Jehenson, Yvonne, «The Pastoral Episode in Cervantes' Don Quijote: Marcela Once Again*, Cervantes, X, 2, 1990, 15-35.

Johnson, Carroll B., Madness and Lust. A Psychoanalitical Approach to "Don Quixote". Berkeley, University of California Press, 1983.

-, "Psychoanalysis and Don Quixote", Richard Bjornson (ed.), Approaches to Teaching Cervantes' "Don Quixote". New York, The Modern Language Association, 1984, 104-12.

-, «People, Real and Fictional», "Don Quixote". The Quest for Modern Fiction. Boston, Twayne, 1990, 104-20.

-. «La sexualidad en el Quijote», Edad de Oro, IX, 1990, 137-48.

Joly, Monioue, «El erotismo en el Quijote: la voz femenina», Edad de Oro, IX, 1990, 125-36.

- «Erotismo y marginación social en la novela cervantinan, Cervantes, XII, 2, 1992, 7-19.

Joset, JACQUES, «De la familia de don Quijote y de la sobrina de éste o 'Familles, je vous hais!' (André Gide)». Actas del II Coloquio Internacional de la Asociación de Cervantistas. Barcelona, Anthropos, 1991, 123-33.

LAMB, RUTH S., «Las mujeres en el Quijote: contraste entre la mujer renacentista y la mujer barrocaw, Manuel Criado de Val (ed.), Cervantes, su obra y su mundo. Actas del I Congreso Internacional sobre cervantes. Madrid, EDI-6. 1991, 767-72.

Madariaga, Salvador de, Guia del lector del "Quijote", [1923]. Buenos Aires, Editorial Sudamericana, 1972, 7." ed.

Maeztu, Ramiro DE, Don Quijote o el amor. [1926]. Edición de Alberto Sánchez. Salamanca, Anaya, 1964.

Mahieu, Roma (sel. y ed.), Amor y sexo en Cervantes. Madrid, Altalena, 1981.

MArouez, héctor P., La representación de los personajes femeninos en el Quijote. Madrid, José Porrúa Turanzas, 1990. 
Molno, Mauricio, «Doña Sancho (Quijote, II, 60)», Homenaje a José Manuel Blecua. Madrid, Gredos, 1983, 443-8.

- «Para una lectura psicológica de los cuentecillos de locos del segundo Quijote., Cervantes, XI, 1, 1991, 87-98.

-, Erratas, Cervantes, XI, 2, 1991, 111.

Natoli, Joseph, y Rusch, Frederix L., Psychocriticism. An Annotated Bibliography. Wesport, Conneticut, Greenwood Press, 1984.

Paiewonsky-CONDE, Edgar, *Cervantes y la teoría reacentista del deseo», ACer, XXIII, 1985, 71-81.

Pini Moro, Donatella, «El Quijote y los dobles: sugerencias para una relectura de la novela cervantina», Actas del I Coloquio de la Asociación de Cervantistas. Barcelona, Anthropos, 1990, 223-33.

Profeti, Marfa Grazia, «Literatura y estudio biográfico. Psicoanálisis y literatura , José María Díez Borque (coord.), Métodos de estudio de la obra literaria. Madrid, Taurus, 1989, reimpresión, 313-52.

Ramón y Cajal, Santiago, "La psicología de Don Quijote de la Mancha y el quijotismo», VV. AA., Discursos leídos en la sesión solemne que el Colegio de Médicos de la provincia de Madrid dedica a Miguel de Cervantes. Madrid, Imprenta de J. A. García, 1905, 37-59.

REDondo, Agustin, «Las dos caras del erotismo en la primera parte del Quijote*, Edad de Oro, IX, 1990, 251-69.

Rey Hazas, Antonio, *Cervantes, el Quijote y una poética de la libertad», Actas del l Coloquio Intermacional de la Asociación de Cervantistas. Barcelona, Anthropos, 1990, 369-80.

Riley, E. C., *Cervantes y Freud*, Insula, 538, octubre, 1991, 34-5.

-, «Cervantes, Freud, and Psychoanalytic Narrative Theory», Modern Language Review, 88, 1, january, 1993, 1-14.

Robert, Marthe, L'ancien et le nouveau. De "Don Quichotte" d Franz Kafka. Paris, Grasset, 1962.

Rodrtguez, Alfred y Rowe, Karl Roland, «Midsummer Eve and the Disenchantment of Dulcineaw, Cervantes, IV, 1, 1984, 79-83.

-, «Cervantes' Redundant Midsummer in Part II of the Quijote», Cervantes, V, 2, 1985, 163-7.

Romera Castillo, Jose, *Don Quijote como alter ego de Cervantes*, Manuel Criado de Val (ed.), Cervantes, su obra y su mundo. Actas del I Congreso Intemacional sobre Cervantes. Madrid, EDI-6, 1981, 493-9.

Rosenblat, Angel, La lengua del "Quijote". Madrid, Gredos, 1971.

RUTKOWSKI, RAINER, «Misoginia y nostalgia en las escenas bucólicas de Quijote*, Cuademos Hispanoamericanos, 430, abril, 1986, 53-62.

TER Host, ROBERT, *On the Importance of Being Earnest: A Reply to Cesáreo Banderaw, Cervantes, V, 1, 1985, 59-63.

Toro Garland, Fernando de, «Aproximación a lo sexual en las Novelas ejemplares de Cervantes*, Manuel Criado de Val (ed.), Cervantes, su obra y su mundo. Actas del I Congreso Internacional sobre Cervantes. Madrid, EDI-6, 1981, 365-70.

VRANICH, S. B., «Sigmund Freud and 'The Case History of Berganza': Freud's Psychoanalytic Beginnings*, Psychoanalytic Review, 63, 1976, 73-82.

Weiger, John G., The Individuated Self: Cervantes and the Emergence of the Individual. Athens, Ohio, Ohio University Press, 1979.

Wright, Elizabeth, Psychoanalytic Criticism: Theory in Practise. Londres y Nueva York, Methuen, 1984. 